\title{
Índices de capacidade para processos multivariados independentes: extensões dos índices de Niverthi e Dey e Mingoti e Glória
}

\author{
Sueli Aparecida Mingotia,", Fernando Luiz Pereira de Oliveira ${ }^{\mathrm{b}}$, \\ Míriam Márcia Carvalho da Conceição ${ }^{c}$ \\ a,"sueliam@est.ufmg.br, UFMG, Brasil \\ bfernandoluizoliveira@ufmg.br, UFMG, Brasil \\ 'miriammcarvalho@uol.com.br, UFMG, Brasil
}

\begin{abstract}
Resumo
Neste artigo apresentamos dois novos índices de capacidade multivariados para processos independentes que são extensões do coeficiente de capacidade univariado $C_{p m}$. Esses novos índices foram formulados de acordo com as ideias de Niverthi e Dey (2000) e Mingoti e Glória (2008) e são sensiveis a diferenças entre as médias de especificação e de processo. Uma comparação entre os índices foi realizada considerando-se vários cenários diferentes e mostrou que o novo coeficiente construído com base na formulação de Mingoti e Glória teve um desempenho melhor que o fundamentado na formulação de Niverthi e Dey, embora ambos tenham se mostrado adequados para avaliar a capacidade de processos multivariados não centrados. Para construção de intervalos de confiança para o índice de capacidade real do processo foi empregada a metodologia Bootstrap. A qualidade dos estimadores dos coeficientes de capacidade foi avaliada por simulação de Monte Carlo.
\end{abstract}

Palavras-chave

Processos multivariados independentes. Índices de capacidade. Normalidade multivariada. Intervalos de confiança. Bootstrap.

\section{Introdução}

0 controle estatístico de processos (CEP) é elemento imprescindível para a sobrevivência e crescimento das empresas na economia moderna. Atualmente as empresas buscam por uma posição competitiva no mercado através do aumento da produtividade e da qualidade de seus produtos. Produzir com qualidade significa ter um processo de fabricação estável.

Uma das abordagens dadas ao termo qualidade é: "[...] qualidade como adequação ao uso do usuário [...]", como dizem Juran e Gryna (1992, p. 51). Essa abordagem parte do princípio de que a qualidade está na capacidade do produto atender a determinadas necessidades do cliente, ou seja, de atender às especificações exigidas pelo cliente (consumidor) do produto/serviço. Desse modo, é necessário implantar mecanismos de monitoramento do processo e medidas que quantifiquem sua capacidade em atender as exigências dos clientes a que seus produtos (efeitos) se destinam. Cartas de controle são úteis para o monitoramento de parâmetros que definem as estruturas de média e variabilidade dos processos, sejam eles univariados ou multivariados (MONTGOMERY, 2004; MASON; YOUNG, 2002). No entanto, não conseguem por si só avaliar se um processo está operando em conformidade com os limites de especificação determinados para o mesmo. Assim, surge a necessidade de métodos específicos para tal quantificação. Em caso de processos univariados, a capacidade do processo pode ser medida através dos coeficientes de capacidade conhecidos como $C_{p}$, $C_{p k}$ e $C_{p m}$ (COSTA; EPPRECHT; CARPINETTI, 2005). Os valores numéricos desses coeficientes estão associados a uma probabilidade de que o processo gere itens não conformes em relação aos limites de especificação. Desse modo, torna-se possível estabelecer faixas de valores para esses índices que 
possam ser utilizadas para a classificação de processos como "altamente capazes" (processos verdes), "razoavelmente capazes" (processos amarelos) e “incapazes” (processos vermelhos). É muito comum, no entanto, que a qualidade de um processo seja determinada por mais de uma característica, sendo então desejável avaliar a capacidade do processo por medidas que levem em consideração todas as características simultaneamente. Uma solução possível é avaliar a capacidade do processo multivariado através do cálculo dos índices $C_{p}, C_{p k} \mathrm{e}$ $C_{p m}$ para cada uma das variáveis separadamente. No entanto, essa solução não leva em consideração a possível correlação existente entre as características de qualidade. Nesse contexto, surgem os índices de capacidade para processos multivariados como os de Chen (1994), Veevers (1998), Niverthi e Dey (2000), Mingoti e Glória (2008), Bernardo e Irony (1998), dentre outros (KOTZ; JOHNSON, 2002; SOARES, 2006). Em linhas gerais, a proposta desses índices é a de estender os coeficientes $C_{p}$ e $C_{p k}$ do caso univariado para a situação multivariada. Outra alternativa ainda não muito explorada na literatura é a extensão do índice univariado $C_{p m}$ para processos multivariados.

Neste artigo são propostos dois novos índices fundamentados nas proposições de Niverthi e Dey (2000) e Mingoti e Glória (2008) e que são extensões do índice $C_{p m}$ univariado para o caso multivariado. A ideia central é construir índices multivariados que levem em consideração a diferença entre os vetores de médias do processo e de especificação, a estrutura de variabilidade e de correlação das características de qualidade do processo e os limites de especificação de cada variável.

Para facilitar o entendimento do conteúdo deste artigo, apresentamos na seção 2 os índices de capacidade multivariados convencionais, isto é, da média geométrica, de Niverthi e Dey (2000) e Mingoti e Glória (2008). Na seção 3 têm-se os dois novos índices propostos neste artigo com um exemplo de cálculo mostrado na seção 4. Na seção 5 apresentam-se os resultados de uma comparação teórica entre os dois índices de capacidade com os convencionais da seção 2. Na seção 6 faz-se uma breve apresentação dos estimadores não viciados dos parâmetros de processos normais multivariados independentes e que são necessários para a estimação dos índices de capacidade. A qualidade de estimação dos coeficientes de capacidade é avaliada na seção 7 através de simulações Monte Carlo. Finalmente, com o intuito de construir intervalos de confiança para os índices de capacidade populacionais, apresenta-se na seção 8 um exemplo empregando-se a metodologia Bootstrap.

\section{Coeficientes de capacidade multivariados}

Os índices de capacidade multivariados que serão apresentados nesta seção estão fundamentados no modelo de probabilidade dado a seguir.

Seja $X=\left(X_{1} X_{2} \ldots X_{p}\right)$ 'o vetor aleatório constituído das $p$ características de qualidade de interesse. Suponha que o vetor $X$ tenha distribuição normal $p$-variada com vetor de médias $\mu^{0}=\left(\mu_{1}^{0} \mu_{2}^{o} \ldots \mu_{p}^{o}\right)^{\prime}$, e matrizes de covariâncias e de correlação denotadas por $\Sigma_{p x p}$ e $P_{p x p}$, respectivamente, sendo essas matrizes positivas definidas (JOHNSON; WICHERN, 2002). Nesse caso, cada variável aleatória $X_{i}$ tem distribuição normal univariada com média $\mu^{0}$, e desvio padrão $\sigma_{i}, i=1,2, \ldots, p$. Sejam $L S L_{i}$ e $U S L_{i}$ os limites inferior e superior de especificação da variável $X_{i}$ respectivamente, $i=1,2, \ldots, p$. Sejam ainda $U S L=\left(\begin{array}{lllll}U S L_{1} & U S L_{2} & \ldots & U S L_{p}\end{array}\right)^{\prime} \mathrm{e}$ $L S L=\left(L S L_{1} L S L_{2} \ldots L S L_{p}\right)^{\prime}$ os vetores contendo os limites de especificação superior e inferior de todas as $p$-variáveis.

\subsection{A média geométrica dos índices de capacidade univariados}

Uma forma simples de avaliar a capacidade de processos multivariados é através da junção das informações obtidas dos índices de capacidade univariados calculados para cada variável $X_{i}, i=1,2, \ldots, p$, separadamente. Essa junção pode ser feita via média geométrica, sendo então definidos os índices multivariados dados pelas Equações 1 e 2:

$$
\begin{aligned}
& C_{\text {pgeom }}=\left(\prod_{i=1}^{p} C_{p}\left(X_{i}\right)\right)^{1 / p} \\
& C_{\text {pkgeom }}=\left(\prod_{i=1}^{p} C_{p k}\left(X_{i}\right)\right)^{1 / p}
\end{aligned}
$$

sendo $C_{p}\left(X_{j}\right)$ e $C_{p k}\left(X_{j}\right)$ os índices de capacidade univariados definidos como:

$$
\begin{aligned}
& C_{p}\left(X_{i}\right)=\frac{U S L_{i}-L S L_{i}}{2 m \sigma_{i}} \\
& C_{p k}\left(X_{i}\right)=\min \left\{\frac{U S L_{i}-\mu_{i}^{0}}{m \sigma_{i}} ; \frac{\mu_{i}^{0}-L S L_{i}}{m \sigma_{i}}\right\}
\end{aligned}
$$

Os índices definidos pela Equação 3, quantificam a relação entre os limites naturais do processo e os limites de especificação. A escolha da constante $m$ é baseada na distribuição normal univariada padronizada, sendo $m=3$ um valor muito comum 
e que corresponde a um nível de confiança igual a 99,73\%, ou um nível de significância $\alpha=0,0027$. 0 nível de significância em questão é aquele utilizado para o controle das características de qualidade quando se consideram gráficos de controle univariados, ou seja, corresponde ao percentual de alarmes falsos estabelecido para a construção do gráfico de controle quando se considera o processo estável, ou sob controle estatístico. Para cada variável o valor de $m$ corresponde à diferença entre o limite superior do processo e a média da variável em questão (ou simetricamente a diferença entre a média e o limite inferior do processo), diferença essa medida em número de desvios padrão.

Embora o uso da média geométrica seja uma forma simples de estender os coeficientes de capacidade univariados para o caso multivariado, os índices nas Equações 1 e 2 não levam em consideração a correlação entre as características de qualidade $X_{i}$. Além disso, como o índice univariado $C_{p k}\left(X_{i}\right)$ definido na Equação 3 pode assumir valores negativos, a média geométrica na Equação 2 nem sempre é matematicamente definida.

\subsection{Indices de capacidade multivariados de Niverthi e Dey}

Em 2000, Niverthi e Dey propuseram uma extensão dos índices de capacidade univariados $C_{p}, C_{p k}$ para o caso multivariado. Esses índices são vetores de dimensão $p \times 1$, sendo que cada coordenada representa $o$ valor do índice de capacidade correspondente a uma das características de qualidade $X_{i}$ do processo. 0 índice de capacidade para cada $X_{i}$ é uma combinação linear das amplitudes dos limites de especificação das $p$ características de qualidade avaliadas. A matriz de ponderação que contém os pesos usados na composição das combinações lineares é a matriz $\Sigma^{-1 / 2}$. Os índices $C_{p}$ e $C_{p k}$ de Niverthi e Dey são definidos pelas Equações 4 e 5.

$$
\begin{aligned}
& C_{p N D}=\frac{1}{2 m} \Sigma^{\frac{-1}{2}}(U S L-L S L) \\
& C_{p k N D}=\Sigma^{\frac{-1}{2}} \min \left[\left(\left(\frac{U S L-\mu^{0}}{m}\right)\right) ;\left(\left(\frac{\mu^{0}-L S L}{m}\right)\right)\right]
\end{aligned}
$$

sendo $\Sigma^{-1 / 2} \Sigma^{-1 / 2}=\Sigma^{-1}$. Na Equação 5 geram-se dois vetores de $p$-coordenadas, e o mínimo é calculado para cada coordenada comparando-se os respectivos valores desses dois vetores, tendo-se, portanto, um índice de capacidade para cada característica de qualidade.
Assim como no caso da média geométrica, a escolha da constante $m$ é baseada na distribuição normal univariada padronizada. Niverthi e Dey (2000) utilizaram $m=3$, valor que corresponde a um nível de confiança igual a 99,73\%. A dificuldade em se utilizarem os índices $C_{p N D}$ e $C_{p k N D}$ para tomar uma decisão sobre a capacidade global do processo vem do fato de que não há um valor de referência que possa ser usado para comparação com os valores produzidos por esses índices. Uma possibilidade é adotar os valores de referência univariados de $C_{p}$ e $C_{p k}$ como 1,33 ou 2, por exemplo, para cada variável separadamente. Outra possibilidade é definir o valor de capacidade global do processo como o valor mínimo das $p$ coordenadas do vetor $C_{p N D}$ se o processo for centrado no vetor de médias nominal, ou como o valor mínimo das $p$ coordenadas do vetor $C_{p k N D}$ se o processo não for centrado (MINGOTI; GLÓRIA, 2008).

\subsection{Indices de capacidade multivaridados de Mingoti e Glória}

Mingoti e Glória (2008) desenvolveram alguns indices de capacidade multivariados chamados de $C_{p}^{m}$ e $C_{p k}^{m}$ que são modificações do coeficiente proposto por Chen (1994) e estão fundamentados nas ideias apresentadas por Hayter e Tsui (1994) no artigo em que esses autores tratavam de um novo teste de hipótese para avaliação de vetores de médias de processos multivariados. Sejam $L S L_{i} \mathrm{e}$ $U S L_{i}$ os limites inferior e superior de especificação da característica de qualidade $X_{i}$. Sejam $r_{i}^{l}=\mu_{i}^{S}-L S L_{i}$ e $r_{i}^{2}=U S L_{i}-\mu_{i}^{S}$, onde $\mu_{i}^{S}$ é a média nominal (especificação) de $X_{i}, i=1,2, \ldots, p$. Então o índice de capacidade multivariado $C_{p}^{m}$ de Mingoti e Glória é definido pelas Equações 6 e 7 como:

$$
C_{p}^{m}=\min \left\{C_{p i}^{m}, i=1,2, \ldots, p\right\}
$$

sendo

$$
C_{p i}^{m}=\left[\frac{U S L_{i}-L S L_{i}}{2 \sigma_{i} C_{r \alpha}}\right]=\left[\frac{r_{i}^{2}+r_{i}^{1}}{2 \sigma_{i} C_{r \alpha}}\right], i=1,2, \ldots, p .
$$

Valores de $C_{p}^{m}$ maiores ou iguais a 1 indicam que o processo é capaz a um nível de confiança de (1- $\alpha)$ $100 \%$. Para um nível de significância $\alpha$ fixo o valor da constante $C_{r \alpha}$ é o ponto crítico usado no teste estatístico de Hayter e Tsui (1994) para o vetor de médias populacional. 0 valor de $C_{r \alpha}$ define a região crítica para rejeição da hipótese nula, $H_{o}: \mu=\mu_{o}$, sendo a hipótese alternativa $H_{a}: \mu \neq \mu_{0}$, onde $\mu_{0}$ é 0 vetor de médias estabelecido na hipótese nula para o processo. De acordo com o teste de Hayter e Tsui, a hipótese nula é rejeitada se a Equação 8 for: 


$$
M=\max \left\{\left|Z_{j}\right|, j=1,2, \ldots, p\right\}>C_{r \alpha}
$$

sendo as variáveis $Z_{j}$ definidas como a Equação 9:

$$
Z_{j}=\frac{X_{j}-\mu_{j}^{0}}{\sigma_{j}}, j=1,2, \ldots, p
$$

onde $\mu_{j}^{o}$ e $\sigma_{j}$ são respectivamente a média e o desvio padrão da variável $X_{j}$ sob a hipótese nula. É importante observar que quando o vetor aleatório $X=\left(X_{1} X_{2} \ldots X_{p}\right)^{\prime}$ 'tem distribuição normal $p$-variada com vetor de médias $\mu^{0}$ e matriz de covariâncias $\Sigma_{p x p}$, o vetor aleatório com as variáveis padronizadas, isto é $Z=\left(Z_{1} Z_{2} \ldots Z_{p}\right)$ ', tem distribuição normal p-variada com vetor de médias nulo e matriz de covariâncias $P_{p x p}$, que por sua vez é a matriz de correlação do vetor aleatório $X$ cujas coordenadas são as características de qualidade avaliadas.

A constante $C_{r \alpha}$ é obtida através de um algoritmo (Quadro 1) que envolve a seleção de amostras aleatórias de uma distribuição normal $p$-variada com vetor de médias nulo e matriz de covariâncias $P_{p x p}$. Quando a matriz $P_{p x p}$ não está previamente especificada, ela pode ser estimada através da matriz de correlação amostral $R_{p x p}$ utilizando dados amostrais do vetor aleatório $X$. Desse modo, o valor crítico $C_{r \alpha}$ é obtido por um procedimento que busca incorporar a correlação entre as características de qualidade para determinação da região de rejeição da hipótese nula. Maiores detalhes técnicos sobre a construção dos coeficientes nas Equações 6 e 7 podem ser encontrados em Mingoti e Glória (2008).

0 coeficiente de capacidade $C_{p}^{m}$ definido na Equação 6 não é sensível a mudanças no vetor de médias do processo em relação ao vetor de médias de especificação. Sendo assim, Mingoti e Glória (2008) propuseram um coeficiente mais adequado para representar essas situações e que é chamado de $C_{p k}^{m}$ dado pela Equação 10:

$C_{p k}^{m}=\min \left\{C_{p k i}^{m}, i=1,2, \ldots, p\right\}$ sendo na Equação 11:

$$
C_{p k i}^{m}=\min \left(\frac{\mu_{i}^{0}-L S L_{i}}{\sigma_{i} C_{r \alpha}} ; \frac{U S L_{i}-\mu_{i}^{0}}{\sigma_{i} C_{r \alpha}}\right), i=1,2, \ldots, p
$$

sendo $\mu_{i}^{o}$ e $\sigma_{i}$, a média e desvio padrão da variável $X_{i}$, respectivamente. Considerando $L S L_{i}=\mu_{i}^{S}-r_{i}^{1} \mathrm{e}$ $U S L_{i}=\mu_{i}^{S}+r_{i}^{2}$, como anteriormente, a Equação 11 pode ser escrita como (Equação 12):

$$
C_{p k i}^{m}=\min \left(\frac{\left(\mu_{i}^{0}-\mu_{i}^{S}+r_{i}^{1}\right)}{\sigma_{i} C_{r \alpha}} ; \frac{\left(\mu_{i}^{S}-\mu_{i}^{0}+r_{i}^{2}\right)}{\sigma_{i} C_{r \alpha}}\right), i=1,2, \ldots, p
$$

o que mostra que $C_{p k}^{m}$ é função da diferença entre as médias do processo e as médias nominais. Para um processo centrado no vetor de médias nominal, o coeficiente $C_{p k}^{m}$ terá o mesmo valor que $C_{p}^{m}$ dado na Equação 6. Os índices de Mingoti e Glória quantificam de uma forma global a capacidade do processo multivariado como também a capacidade relativa a cada variável individualmente já que é o mínimo de um conjunto cujos componentes são os coeficientes de capacidade de cada variável $X_{i}, i=1,2, \ldots, p$, separadamente.

\section{Extensão dos índices de capacidade de Niverthi e Dey e Mingoti e Glória}

Considere o índice de capacidade univariado $C_{p m_{j}}$ da característica de qualidade $X_{i}$ definido na Equação 13 , para um nível de confiança de $(1-\alpha) 100 \%$ :

$$
C_{p m_{i}}=C_{p m}\left(X_{i}\right)=\frac{U S L_{i}-L S L_{i}}{2 m \sqrt{\sigma_{i}^{2}+\left(\mu_{i}^{S}-\mu_{i}^{0}\right)^{2}}}, i=1,2, \ldots, p
$$

sendo $m$ a constante proveniente da distribuição normal padronizada como tratado na seção 2 . 0 índice $C_{p m_{i}}$ dado na Equação 13 pode ser reescrito da seguinte forma na Equação 14:

$$
C_{p m_{i}}=C_{p m}\left(X_{i}\right)=\frac{U S L_{i}-L S L_{i}}{2 m \sqrt{E\left[\left(X_{i}-\mu_{i}^{S}\right)^{2}\right]}}, i=1,2, \ldots, p
$$

Quadro 1. Algoritmo para determinação da constante $C_{r \alpha}$.

Passo 1. Gerar um grande número $N$ de vetores de observações de uma distribuição normal $p$-variada com vetor de médias zero e matriz de covariâncias $P_{p x p}$. Denote esses vetores por: $Z^{1}, Z^{2}, \ldots Z^{N}$.

Passo 2. Calcule a estatística $M$ para cada um dos $N$ vetores $Z^{i}=\left(Z_{1}^{i}, Z_{2}^{i}, \ldots Z_{p}^{i}\right)^{\prime}, i=1,2, \ldots, N$, gerados no passo 1 , isto é, calcule:

$$
M^{i}=\max \left\{\left|Z_{j}^{i}\right|, j=1,2, \ldots, p\right\}
$$

Passo 3. Obtenha a distribuição empírica da estatística $M$ utilizando os vetores, encontre a ordenada correspondente ao quantil de ordem $(1-\alpha)$ e utilize esse valor como o correspondente a constante crítica, $C_{r \alpha}, 0<\alpha<1$. 
Sendo $\mu_{i}^{s}$ a média de especificação da característica de qualidade $X_{i}$ e $E\left[\left(X_{i}-\mu_{i}^{s}\right)^{2}\right]$ a esperança matemática da variável $\left(X_{i}-\mu_{i}^{S}\right)^{2}$. Pode ser mostrado na Equação 15 que:

$$
E\left[\left(X_{i}-\mu_{i}^{S}\right)^{2}\right]=\sigma_{i}^{2}+\left[\left(\mu_{i}^{0}-\mu_{i}^{S}\right)^{2}\right]
$$

o que em Estatística, representa o erro quadrático médio. Portanto o índice $C_{p m}\left(X_{i}\right)$ nada mais é do que o índice $C_{p}$ univariado com o denominador substituído pela Equação 15. Sendo assim, o desvio padrão do processo que aparece no denominador do índice $C_{p}$, e que por sua vez é obtido considerando-se a média do processo como referência, é substituído por um valor de variabilidade calculado tendo-se como referência o valor da média de especificação da variável.

Assim como o coeficiente univariado $C_{p k}\left(X_{i}\right)$ descrito na seção 2.1 , o coeficiente $C_{p m}\left(X_{i}\right)$ é sensível a desvios entre as médias de especificação e do processo da variável $X_{i}$. Levando isso em consideração torna-se natural procurar estender os coeficientes multivariados de Niverthi e Dey (2000) e Mingoti e Glória (2008) utilizando-se o mesmo princípio de construção do coeficiente univariado $C_{p m}\left(X_{i}\right)$. Desse modo, propomos dois novos índices de capacidade multivariados que serão chamados respectivamente de $C_{p m A}^{M}$ e $C_{p m B}^{M}$. 0 primeiro é uma versão multivariada de $C_{p m}\left(X_{i}\right)$ seguindo a formulação de índice de Niverthi e Dey (2000), enquanto o segundo é a versão de acordo com a formulação de Mingoti e Glória (2008). 0 índice $C_{p m A}^{M}$ é definido pela Equação 16:

$C_{p m A}^{M}=\left(\Sigma_{p x p}+A\right)^{-1 / 2}\left[\frac{U S L-L S L}{2 m}\right]$

onde $A=\left(\mu^{S}-\mu^{0}\right)\left(\mu^{S}-\mu^{0}\right)$ ' é uma matriz de dimensão $p \times p$ que está relacionada com os desvios das médias de especificação e de processo de cada característica de qualidade, sendo $\mu^{s}=\left(\mu_{1}^{s} \mu_{2}^{s} \ldots \mu_{p}^{s}\right)^{\prime}$ e $\mu^{o}=\left(\mu_{1}^{o} \mu_{2}^{o} \ldots \mu_{p}^{o}\right)$ : 0 valor da constante $m$, definido como na seção 2 , é proveniente da distribuição normal univariada padronizada e seu valor é escolhido de acordo com o nível de confiança que o usuário deseja utilizar para medir a capacidade do processo. Para processos centrados nas médias de especificação o valor de $C_{p m A}^{M}$ será o mesmo que o dos coeficientes $C_{p N D}$ e $C_{p k N D}$ definidos na seção 2.2.

Para melhor entender a motivação da construção da Equação 16 podemos nos reportar novamente ao conceito de erro quadrático médio, estendendo-o para o caso multivariado. Seja $X=\left(X_{1} X_{2} \ldots X_{p}\right)^{\prime}$ 'um vetor aleatório. Então a matriz de covariâncias $\Sigma_{p \chi p}$ da distribuição de probabilidades do vetor $X$ pode ser expressa na Equação 17 como:

$$
\begin{aligned}
\operatorname{Var}(X)= & \Sigma_{p \times p}=E\left[\left(X-\mu^{0}\right)\left(X-\mu^{0}\right)^{\prime}\right] \\
& =E\left[\left(X-\mu^{s}-\mu^{0}+\mu^{s}\right)\left(X-\mu^{s}-\mu^{0}+\mu^{s}\right)^{\prime}\right] \\
& =E\left[\left(X-\mu^{s}\right)\left(X-\mu^{s}\right)^{\prime}\right]-E\left[\left(\mu^{s}-\mu^{0}\right)\left(\mu^{s}-\mu^{0}\right)^{\prime}\right] \\
& =E\left[\left(X-\mu^{s}\right)\left(X-\mu^{s}\right)^{\prime}\right]-A_{p \times p}
\end{aligned}
$$

Desse modo, tem-se a relação:

$$
E\left[\left(X-\mu^{S}\right)\left(X-\mu^{S}\right)^{\prime}\right]=\Sigma_{p x p}+A_{p x p}
$$

sendo assim, a ideia é substituir a matriz de covariâncias $\Sigma$ que aparece na Equação 4 do índice de capacidade $C_{p N D}$ de Niverthi e Dey pela expressão dada na Equação 18. 0 índice $C_{p m A}^{M}$ assim construído é função de uma medida de variabilidade que tem como referência o vetor de médias de especificação ao invés do vetor de médias do processo.

0 índice $C_{p m B}^{M}$ é definido como na Equação 19, sendo o processo considerado capaz se o valor de $C_{p m B}^{M}$ for maior ou igual a 1. Quando o processo é centrado nas médias de especificação o valor de $C_{\mathrm{pmB}}^{\mathrm{M}}$ será o mesmo que os dos coeficientes $C_{p}^{m} \mathrm{e} C_{p k}^{m}$ definidos na seção 2.3 (Equações 6 e 11).

$C_{p m B}^{M}=\min \left\{C_{p m i B}^{M}, i=1,2, \ldots, p\right\}$

onde a Equação 19:

$$
C_{p m i B}^{M}=\frac{U S L_{i}-L S L_{i}}{2 C_{r \alpha}\left(\sigma_{i}^{2}+\left(\mu_{i}^{s}-\mu_{i}^{0}\right)^{2}\right)^{\frac{1}{2}}}=\frac{r_{i}^{1}+r_{i}^{2}}{2 C_{r \alpha}\left(\sigma_{i}^{2}+\left(\mu_{i}^{s}-\mu_{i}^{0}\right)^{2}\right)^{\frac{1}{2}}}
$$

sendo $C_{r \alpha}, L S L_{i}=\mu_{i}^{S}-r_{i}^{l}$ e $U S L_{i}=\mu_{i}^{S}+r_{i}^{2}$ como definidos na seção 2.3 , o que mostra que o índice $C_{p m B}^{M}$ também leva em consideração a diferença entre os vetores de médias do processo e especificação. A motivação por detrás da Equação 20 é semelhante àquela apresentada para o índice univariado $C_{p m_{i}}$ (Equação 13) e o multivariado $C_{p m A}^{M}$ proposto neste artigo.

\section{Exemplo: cálculo dos índices de capacidade multivariados}

Para ilustrar, mostramos um exemplo de como os indices de capacidade multivariados tratados neste artigo são calculados para $p=2$. As informações necessárias para os cálculos dos índices estão na Tabela 1, sendo a matriz de covariâncias das duas variáveis dada por: $\Sigma=\left[\begin{array}{cc}1 & 0,5 \\ 0,5 & 1\end{array}\right]$, ou seja, a correlação entre as duas variáveis é igual a 0,5. 
Para um nível de confiança de $99,73 \%$ a constante $m=3$ e a constante $C_{r \alpha}$ é igual a 2,906 (valor obtido de acordo com o algoritmo do Quadro 1 considerando-se a matriz de correlação $P_{2 \times 2}$ igual a matriz de covariâncias $\Sigma$ e $N=100000$ ).

(1) $C_{p}$ univariado:

$1{ }^{\text {a }}$ variável: $C_{p}=\frac{50-30}{6}=3,333 \mathrm{e}$

$2^{\text {a }}$ variável: $C_{p}=\frac{38,4-21,59}{6}=2,801$

(2) $C_{p k}$ univariado:

$1^{\text {a }}$ variável: $C_{p k}=\min \left[\frac{50-42}{3} ; \frac{42-30}{3}\right]=\min [2,6664]=2,666$ $2^{2}$ variável: $C_{p k}=\min \left[\frac{38,4-30}{3} ; \frac{30-21,59}{3}\right]=\min [2,82,803]=2,8$

(3) $C_{\text {pgeom }}$ (média geométrica) $=(3,333 \times 2,801)^{\frac{1}{2}}=3,055$

(4) $C_{p k g e o m}$ (média geométrica) $=(2,666 \times 2,8)^{\frac{1}{2}}=2,732$

(5) $C_{p N D}$ (Niverthi e Dey): Primeiramente calcula-se a matriz inversa de: $\Sigma^{-1 / 2}$ :

$\Sigma^{-\frac{1}{2}}=\left[\begin{array}{cc}1,1153 & -0,2988 \\ -0,2988 & 1,1153\end{array}\right] .0$ coeficiente de capacidade de Niverthi e Dey será obtido pela Equação 21:

$C_{P N D}=\Sigma^{-\frac{1}{2}}\left(\begin{array}{c}3,333 \\ 2,801\end{array}\right)=\left(\begin{array}{c}1,1153(3,333)-0,2988(2,801) \\ -0,2988(3,333)+1,1153(2,801)\end{array}\right)=\left(\begin{array}{l}2,880 \\ 2,128\end{array}\right)$

0 valor 2,880 representa a capacidade do processo em relação à $1^{\text {a }}$ variável e 2,128 a capacidade em relação à $2^{\text {a }}$ variável. 0 coeficiente de capacidade global do processo pode ser definido como 2,128 , que é o mínimo entre os dois valores.

(6) $C_{p k N D}$ (Niverthi e Dey)

$C_{p k N D}=\Sigma^{-\frac{1}{2}} \min \left[\left(\left(\begin{array}{c}2,666 \\ 2,800\end{array}\right)\right) ;\left(\left(\begin{array}{c}4 \\ 2,803\end{array}\right)\right)\right]=\Sigma^{-\frac{1}{2}}\left(\begin{array}{l}2,666 \\ 2,800\end{array}\right)=\left(\begin{array}{c}2,137 \\ 2,326\end{array}\right)$

Neste caso, o índice global de capacidade do processo seria 2,137 .

Tabela 1. Parâmetros para o cálculo dos índices de capacidade - Exemplo.

\begin{tabular}{lcc}
\hline \multicolumn{1}{c}{ Parâmetros } & $\begin{array}{c}1^{\text {a }} \\
\text { variável }\end{array}$ & $\begin{array}{c}2^{\text {a }} \\
\text { variável }\end{array}$ \\
\hline Limite superior de especificação (USL) & 50 & 38,40 \\
Limite inferior de especificação (LSL) & 30 & 21,59 \\
Média de especificação (nominal) & 40 & 30 \\
Média do processo & 42 & 30 \\
Desvio padrão do processo & 1 & 1 \\
\hline
\end{tabular}

(7) $C_{p}^{m}$ (Mingoti e Glória)

$$
\begin{aligned}
& C_{p}^{m}=\min \left\{\frac{U S L_{j}-L S L_{j}}{\sigma_{j} C_{r \alpha}}, j=1,2\right\}= \\
& \left\{\frac{50-30}{2(2,906)} ; \frac{38,4-21,59}{2(2,906)}\right\}=\min \{3,441 \quad 2,892\}=2,892
\end{aligned}
$$

É importante observar que por definição o índice de Mingoti e Glória é o mínimo entre os índices de capacidade da primeira e segunda variáveis, sendo assim quando o índice $C_{p}^{m}$ indica que o processo é não capaz, é possível identificar quais são as variáveis responsáveis por tal condição.

(8) $C_{p k}^{m}$ (Mingoti e Glória)

$$
\begin{aligned}
C_{p k}^{m} & =\min \left\{\left(\frac{\mu_{j}^{0}-L S L_{j}}{\sigma_{j} C_{r \alpha}} ; \frac{U S L_{j}-\mu_{j}^{0}}{\sigma_{j} C_{r \alpha}}\right) ; j=1,2\right\} \\
& =\min \left\{\left(\frac{42-30}{2,906} ; \frac{50-42}{2,906}\right) ;\left(\frac{30-21,59}{2,906} ; \frac{38,4-30}{2,906}\right)\right\} \\
& =\min \{(4,129 \quad 2,752) ;(2,894 \quad 2,890)\}=2,752
\end{aligned}
$$

(9) $C_{p m A}^{M}$ (Extensão do $C_{\mathrm{pm}}$ univariado via Niverthi e Dey)

$$
C_{p m A}^{M}=(\Sigma+A)^{-1 / 2}\left[\frac{U S L-L S L}{6}\right] \text {, sendo } A=\left(\begin{array}{ll}
4 & 0 \\
0 & 0
\end{array}\right)
$$

Então:

$C_{p m A}^{M}=\left(\begin{array}{ll}5 & 0,5 \\ 0,5 & 1\end{array}\right)^{\frac{-1}{2}}\left[\begin{array}{l}3,333 \\ 2,801\end{array}\right]=\left(\begin{array}{cc}0,453 & -0,071 \\ -0,071 & 1,023\end{array}\right)\left[\begin{array}{l}3,333 \\ 2,801\end{array}\right]=\left(\begin{array}{l}1,311 \\ 2,629\end{array}\right)$ logo o índice de capacidade global seria igual a 1,311.

(10) $C_{p m B}^{M}$ (Extensão do $C_{p m}$ univariado via Mingoti e Glória)

Tem-se que:

$$
\begin{gathered}
C_{p m 1 B}^{M}=\frac{U S L_{1}-L S L_{1}}{2 \cdot C_{r \alpha}\left(\sigma_{1}^{2}+\left(\mu_{1}^{s}-\mu_{1}^{0}\right)^{2}\right)^{\frac{1}{2}}}=\frac{50-30}{2(2,906)\left(1+(2)^{2}\right)^{\frac{1}{2}}}=1,539 \\
C_{p m 2 B}^{M}=\frac{U S L_{2}-L S L_{2}}{2 C_{r \alpha}\left(\sigma_{2}^{2}+\left(\mu_{2}^{s}-\mu_{2}^{0}\right)^{2}\right)^{1 / 2}}=\frac{38,40-21,59}{2(2,906)\left(1+(0)^{2}\right)^{1 / 2}}=2,892
\end{gathered}
$$

Então: $C_{p m B}^{M}=\min \{1,539 ; 2,892\}=1,539$

Neste exemplo, é possível observar que os novos coeficientes de capacidade $C_{p m A}^{M}$ e $C_{p m B}^{M}$ são mais sensiveis ao deslocamento das médias do processo em relação às médias nominais em comparação com os indices $C_{p k g e o m}, C_{p k N D}$ e $C_{p k}^{m}$. Ambos conseguiram sinalizar de uma forma mais apropriada a não centralidade do processo em relação à primeira variável. 


\section{Comparação entre os índices de capacidade multivariados}

Nesta seção apresentamos uma comparação entre os índices de capacidade multivariados tratados neste artigo. 0 estudo foi feito para $p=2$ e 3 considerando-se vários cenários teóricos diferentes em termos de limites de especificação e de processo como descritos nas Tabelas 2 e 3. Para $p=2$ tem-se 3 processos centrados na média nominal (casos 1,2 , 3), enquanto para $p=3$ tem-se dois (casos 1,2$)$. Em cada situação os coeficientes de capacidade foram calculados a 99,73\% considerando-se as seguintes matrizes de covariâncias para $p=2$ e $p=3$ respectivamente na Equação 22:

$\Sigma_{2 \times 2}=\left[\begin{array}{cc}1 & 0,5 \\ 0,5 & 1\end{array}\right]$ e $\Sigma_{3 \times 3}=\left[\begin{array}{ccc}1 & 0,5 & 0,7 \\ 0,5 & 1 & 0,3 \\ 0,7 & 0,3 & 1\end{array}\right]$

Para o índice de Niverthi e Dey, o coeficiente de capacidade global do processo foi definido como o mínimo entre os valores das $p$ coordenadas dos vetores $C_{p N D}$ e $C_{p k N D}$ respectivamente, de forma similar ao que foi feito no exemplo da seção 4. Os resultados obtidos dos índices de capacidade são mostrados nas Tabelas 4 e 5. Para o caso bivariado são apresentadas também as probabilidades de 0 processo gerar um item não conforme de acordo com as respectivas especificações. Para ilustrar, a região de especificação e a região de confiança (a 99,73\%) dos processos são apresentadas na Figura 1 para os cenários da Tabela $2(p=2)$. Comparando-se os gráficos com os valores dos índices de capacidade correspondentes (Tabela 4), no caso dos processos não centrados é possível ver que, com exceção da média geométrica $\left(C_{\text {pgeom }}\right)$, Niverthi e Dey $\left(C_{p N D}\right)$ e Mingoti e Glória $\left(C_{p}^{m}\right)$, todos os outros coeficientes de capacidade multivariados identificaram corretamente a situação em que o processo era de fato não capaz (casos 4, 6). Para a situação em que o processo era centrado e não capaz (caso 2), apenas a média geométrica não identificou a falta de capacidade do processo. No entanto, há de se ressaltar que os coeficientes de Niverthi e Dey (2000), assim como o índice $C_{p m A}^{M}$ que também está fundamentado na formulação de Niverthi e Dey, tendem a resultar em valores numéricos menores do que os índices fundamentados em Mingoti e Glória (2008) mesmo para processos capazes. 0 caso 3, que é um processo centrado nas médias de especificação, mostra bem essa característica. Pela Figura 1, nota-se que esse processo é capaz e com uma probabilidade de gerar itens não conformes igual a zero. No entanto, os indices de Niverthi e Dey resultam em valores muito menores que 1 , indicando que se tem um processo não capaz. Esse fato é devido à segunda variável que apresenta capacidade inferior à primeira, mas ainda acima do valor de referência 1,33 usado para classificação de processos univariados. Já os indices de Mingoti e Glória resultam em valores maiores que 1 e mais razoáveis, indicando que o processo é capaz. Esse exemplo vem ao encontro do que já foi salientado em Mingoti e Glória (2008) sobre o fato de ser possivel que um processo capaz seja considerado incapaz sob a ótica dos índices de capacidade multivariados fundamentados em Niverthi e Dey (2000).

Tabela 2. Limites de especificação e do processo $-p=2$.

\begin{tabular}{|c|c|c|c|c|c|}
\hline Casos & LE-variável 1 & LE-variável 2 & LP-variável 1 & LP-variável 2 & Tipo de processo \\
\hline 1 & {$\left[\begin{array}{lll}30 & 40 & 50\end{array}\right]$} & {$\left[\begin{array}{llll}21,6 & 30 & 38,4\end{array}\right]$} & {$\left[\begin{array}{lll}37 & 40 & 43\end{array}\right]$} & {$\left[\begin{array}{lll}27 & 30 & 33\end{array}\right]$} & centrado e capaz \\
\hline 2 & {$\left[\begin{array}{lll}30 & 40 & 50\end{array}\right]$} & {$\left[\begin{array}{llll}28,0 & 30 & 32,0\end{array}\right]$} & {$\left[\begin{array}{lll}37 & 40 & 43\end{array}\right]$} & {$\left[\begin{array}{lll}27 & 30 & 33\end{array}\right]$} & centrado e não capaz \\
\hline 3 & {$\left[\begin{array}{lll}30 & 40 & 50\end{array}\right]$} & {$\left[\begin{array}{lll}25,8 & 30 & 34,2\end{array}\right]$} & {$\left[\begin{array}{lll}37 & 40 & 43\end{array}\right]$} & {$\left[\begin{array}{lll}27 & 30 & 33\end{array}\right]$} & centrado e capaz \\
\hline 4 & {$\left[\begin{array}{lll}30 & 40 & 50\end{array}\right]$} & {$\left[\begin{array}{llll}21,6 & 30 & 38,4\end{array}\right]$} & {$\left[\begin{array}{lll}45 & 48 & 51\end{array}\right]$} & [27 30 33 $]$ & não centrado e não capaz \\
\hline 5 & {$\left[\begin{array}{lll}30 & 40 & 50\end{array}\right]$} & {$\left[\begin{array}{llll}21,6 & 30 & 38,4\end{array}\right]$} & [39 42 45] & {$\left[\begin{array}{lll}29 & 32 & 35\end{array}\right]$} & não centrado e capaz \\
\hline 6 & {$\left[\begin{array}{lll}30 & 40 & 50\end{array}\right]$} & {$\left[\begin{array}{llll}21,6 & 30 & 38,4\end{array}\right]$} & {$\left[\begin{array}{lll}45 & 48 & 51\end{array}\right]$} & {$\left[\begin{array}{lll}37 & 40 & 43\end{array}\right]$} & não centrado e não capaz \\
\hline
\end{tabular}

LE: Limites de especificação; LP: Limites de processo.

Tabela 3. Limites de especificação e do processo $-p=3$.

\begin{tabular}{|c|c|c|c|c|}
\hline Parâmetros & Caso 1 & Caso 2 & Caso 3 & Caso 4 \\
\hline Limites de especificação - variável 1 & {$[33,04047,0]$} & {$\left[\begin{array}{lll}33 & 40 & 47\end{array}\right]$} & {$\left[\begin{array}{lll}33 & 40 & 47\end{array}\right]$} & {$\left[\begin{array}{lll}33 & 40 & 47\end{array}\right]$} \\
\hline Limites de especificação - variável 2 & {$\left[\begin{array}{llll}21,6 & 30 & 38,4\end{array}\right]$} & {$\left[\begin{array}{lll}27 & 30 & 33\end{array}\right]$} & {$\left[\begin{array}{llll}21,6 & 30 & 38,4\end{array}\right]$} & {$\left[\begin{array}{llll}21,6 & 30 & 38,4\end{array}\right]$} \\
\hline Limites de especificação - variável 3 & {$\left[\begin{array}{llll}13,6 & 20 & 26,4\end{array}\right]$} & {$\left[\begin{array}{llll}17,8 & 20 & 22,2\end{array}\right]$} & {$\left[\begin{array}{llll}13,6 & 20 & 26,4\end{array}\right]$} & {$\left[\begin{array}{llll}13,6 & 20 & 26,4\end{array}\right]$} \\
\hline Limites do processo - variável 1 & {$\left[\begin{array}{lll}37 & 40 & 43\end{array}\right]$} & {$\left[\begin{array}{lll}37 & 40 & 43\end{array}\right]$} & {$\left[\begin{array}{lll}42 & 45 & 48\end{array}\right]$} & {$\left[\begin{array}{lll}43 & 46 & 49\end{array}\right]$} \\
\hline Limites do processo - variável 2 & {$\left[\begin{array}{lll}27 & 30 & 33\end{array}\right]$} & {$\left[\begin{array}{lll}27 & 30 & 33\end{array}\right]$} & {$\left[\begin{array}{lll}31 & 34 & 37\end{array}\right]$} & {$\left[\begin{array}{lll}32 & 35 & 38\end{array}\right]$} \\
\hline Limites do processo - variável 3 & {$\left[\begin{array}{lll}17 & 20 & 23\end{array}\right]$} & {$\left[\begin{array}{lll}17 & 20 & 23\end{array}\right]$} & {$\left[\begin{array}{lll}20 & 23 & 26\end{array}\right]$} & {$\left[\begin{array}{lll}21 & 24 & 27\end{array}\right]$} \\
\hline Tipo de processo & centrado e capaz & $\begin{array}{l}\text { centrado e } \\
\text { não capaz }\end{array}$ & $\begin{array}{c}\text { não centrado e } \\
\text { não capaz }\end{array}$ & $\begin{array}{c}\text { não centrado e } \\
\text { não capaz }\end{array}$ \\
\hline
\end{tabular}


Tabela 4. Resultados dos coeficientes de capacidade $-p=2$.

\begin{tabular}{ccccccc}
\hline İndices de capacidade & Caso 1 & Caso 2 & Caso 3 & Caso 4 & Caso 5 & Caso 6 \\
\hline$C_{P}-$ variável 1 & 3,33 & 3,33 & 3,33 & 3,33 & 3,33 & 3,33 \\
$C_{P}-$ variável 2 & 2,80 & 0,67 & 1,40 & 2,80 & 2,80 & 2,80 \\
$C_{P k}-$ variável 1 & 3,33 & 3,33 & 3,33 & 0,67 & 2,67 & 0,67 \\
$C_{P k}-$ variável 2 & 2,80 & 0,67 & 1,40 & 2,80 & 2,13 & $-0,53$ \\
$C_{P}$ (geométrica) & 3,05 & 1,49 & 2,16 & 3,05 & 3,05 & 3,05 \\
$C_{P k}$ (geométrica) & 3,05 & 1,49 & 2,16 & 1,37 & 2,38 & n.c. \\
$C_{P}$ (Niverthi-Dey) & 2,13 & $-0,25$ & 0,57 & 2,13 & 2,13 & 2,13 \\
$C_{P k}$ (Niverti-Dey) & 2,13 & $-0,25$ & 0,57 & $-0,10$ & 1,58 & $-0,79$ \\
$C_{p m}$ (Mingoti-Glória) & 2,89 & 0,69 & 1,45 & 2,89 & 2,89 & 2,89 \\
$C_{p k m}$ (Mingoti-Glória) & 2,89 & 0,69 & 1,45 & 0,69 & 2,20 & $-0,55$ \\
$C_{p m A}$ (Niverthi e Dey) & 2,13 & $-0,25$ & 0,57 & 0,39 & 0,62 & $-0,48$ \\
$C_{p m B}$ (Mingoti-Glória) & 2,89 & 0,69 & 1,45 & 0,43 & 1,29 & 0,28 \\
Valor da probabilidade $p$ & 0 & 0,0455 & 0 & 0,02275 & 0 & 0,9464 \\
\hline
\end{tabular}

n.c: não é possível calcular; $p$ é a probabilidade de o processo gerar um item não conforme de acordo com as especificações.

Tabela 5. Resultados dos coeficientes de capacidade $-p=3$.

\begin{tabular}{ccccc}
\hline Índices de capacidade & Caso 1 & Caso 2 & Caso 3 & Caso 4 \\
\hline$C_{P}-$ variável 1 & 2,33 & 2,33 & 2,33 & 2,33 \\
$C_{P}-$ variável 2 & 2,80 & 1,00 & 2,80 & 2,80 \\
$C_{P}-$ variável 3 & 2,13 & 0,73 & 2,13 & 2,13 \\
$C_{P k}-$ variável 1 & 2,33 & 2,33 & 0,66 & 0,33 \\
$C_{P k}-$ variável 2 & 2,80 & 1,00 & 1,47 & 1,13 \\
$C_{P k}-$ variável 3 & 2,13 & 0,73 & 1,13 & 0,80 \\
$C_{P}$ (geométrica) & 2,41 & 1,20 & 2,41 & 2,41 \\
$C_{P k}$ (geométrica) & 2,41 & 1,20 & 1,03 & 0,67 \\
$C_{P}$ (Niverthi-Dey) & 1,33 & $-0,30$ & 1,33 & 1,33 \\
$C_{P k}$ (Niverti-Dey) & 1,33 & $-0,30$ & $-0,10$ & $-0,30$ \\
$C_{p m}$ (Mingoti-Glória) & 2,10 & 0,72 & 2,03 & 2,03 \\
$C_{p k m}$ (Mingoti-Glória) & 2,10 & 0,72 & 0,63 & 0,32 \\
$C_{p m A}$ (Niverthi-Dey) & 1,33 & $-0,30$ & $-0,66$ & $-0,61$ \\
$C_{p m B}$ (Mingoti-Glória) & 2,10 & 0,72 & 0,44 & 0,36 \\
\hline
\end{tabular}

A penalização de Niverthi e Dey é ainda maior para processos que apresentam qualquer tipo de desvio da média do processo em relação à média nominal. Comparando-se apenas os índices $C_{p m A}^{M}$ e $C_{p m B}^{M}$ vê-se que ambos penalizam processos que não estão centrados nas médias nominais, mas o segundo índice apresenta resultados mais razoáveis que o primeiro. A mesma tendência é observada quando se aumenta o número de variáveis para $p=3$. É possível ver também que os índices da média geométrica $C_{p g e o m}$, assim como os índices de Niverthi e Dey $\left(C_{p N D}^{p g e o m}\right)$ e Mingoti e Glória $\left(C_{p}^{m}\right)$, só devem ser utilizados em situações nas quais o processo é centrado no vetor de médias nominal.

\section{Estimativa dos índices de capacidade multivariados}

$\mathrm{Na}$ prática os índices de capacidade são estimados a partir de amostras do processo. Suponha que se tenha observado $Q$ subgrupos independentes de tamanhos $n, n \geq 1$, do vetor aleatório $X$ que contém as características de qualidade de interesse, $X$ com distribuição normal $p$-variada com vetor de médias $\mu^{o}$ e matriz de covariâncias $\Sigma$. Seja $n>1 \mathrm{e}$ $\bar{X}_{\mathrm{k}}$ e $S_{\mathrm{k}}$ o vetor de médias e a matriz de covariâncias amostrais do subgrupo $k$, respectivamente. Nesse caso, as estimativas não viciadas do vetor de médias do processo $\mu^{0}$ e da matriz de covariâncias $\Sigma$ são dadas por $\overline{\bar{X}}$ e $\bar{S}$ definidas pela Equação 23 (MONTGOMERY, 2004):

$$
\overline{\bar{X}}=\frac{1}{Q} \sum_{k=1}^{Q} \bar{X}_{k} ; \bar{S}=\frac{1}{Q} \sum_{k=1}^{Q} S_{k}
$$

No caso em que $n=1$ (observações individuais) o vetor de médias do processo é estimado pelo vetor de médias amostral calculado considerando as $Q$ observações amostrais em conjunto. A matriz de covariâncias $\Sigma$ por sua vez pode ser estimada pela matriz de covariâncias amostral calculada com base nas $Q$ observações coletadas ou pela matriz de diferenças sucessivas das $Q$ observações amostrais, método este que é semelhante ao da amplitude móvel usado no caso univariado para controle da variabilidade do processo (MONTGOMERY, 2004). Para exemplificar, sejam $X_{1}, X_{2} \ldots X_{Q}$ os $Q$ vetores amostrais observados do processo, $X_{\mathrm{i}}$ de dimensão $p \times 1, i=1,2, \ldots, Q$, e seja $\bar{X}$ o vetor de médias amostral. A matriz de covariâncias amostral será definida como na Equação 24:

$$
S=\frac{1}{Q-1} \sum_{i=1}^{Q}\left(X_{i}-\bar{X}\right)\left(X_{i}-\bar{X}\right)^{\prime}
$$

Seja 0 vetor de diferenças $v_{i}=\left(X_{i+1}-X_{j}\right)$, $i=1,2, \ldots, Q-1$, e seja $V$ a matriz $p x p$ definida pela Equação 25: 


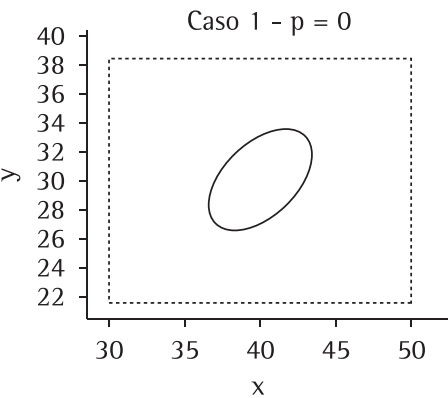

LP-variável 1: [37 40 43]

LP-variável 2: [27 30 33]

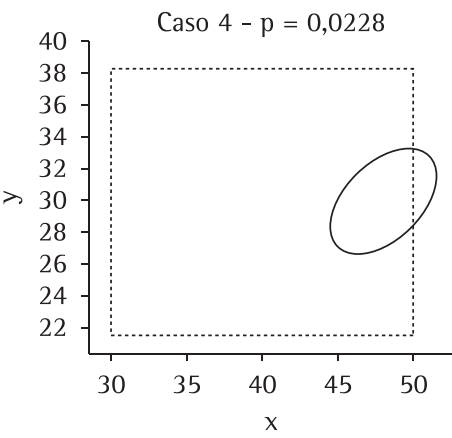

LP-variável 1: [45 48 51]

LP-variável 2: [27 30 33]

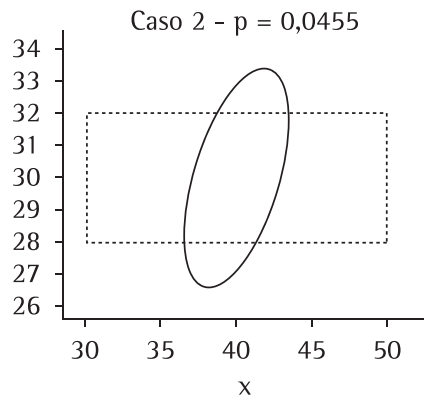

LP-variável 1: [37 40 43]

LP-variável 2: [ [27 30 33]

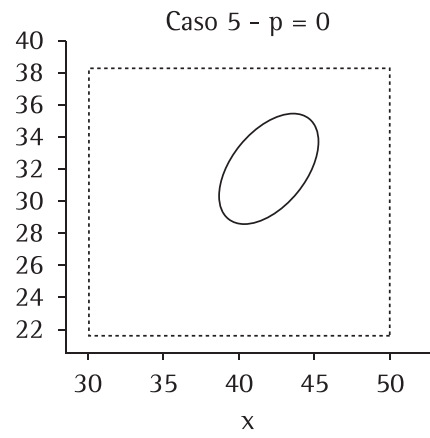

LP-variável 1: [39 42 45] LP-variável 2: [29 32 35]

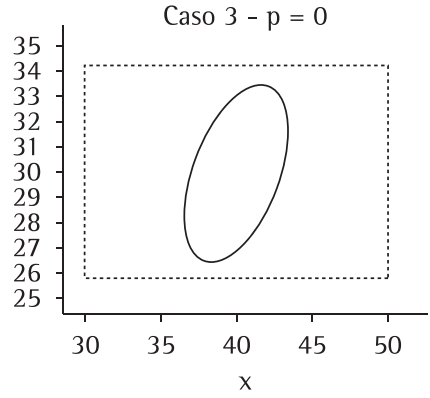

LP-variável 1: [37 40 43]

LP-variável 2: [27 30 33]

Caso $5-p=0,9465$

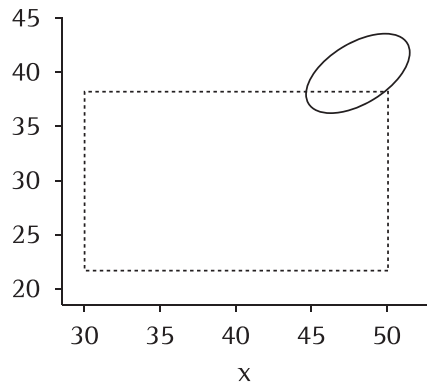

LP-variável 1: [45 48 51]

LP-variável 2: [37 40 43]

Figura 1. Elipses de confiança (99,73\%) e regiões de especificação dos modelos da Tabela 2 juntamente com a probabilidade p de itens não conformes. LP: limites do processo.

$$
V=\left[\begin{array}{c}
v_{1}^{\prime} \\
v_{2}^{\prime} \\
\vdots \\
v_{Q-1}
\end{array}\right]
$$

Então, o estimador de diferenças sucessivas, não viciado, da matriz $\Sigma$ é dado pela Equação 26:

$$
S^{*}=\frac{1}{2(Q-1)} V^{\prime} V
$$

A partir das estimativas do vetor de médias do processo $\mu^{o}$ e da matriz de covariâncias $\Sigma$, todos os índices de capacidade mencionados neste artigo podem ser calculados bastando que se substituam os parâmetros $\mu^{o}$ e $\Sigma$ pelas suas respectivas estimativas em cada fórmula dos índices de capacidade apresentados. A constante $C_{r \alpha}$ necessária para o cálculo dos índices de Mingoti e Glória é obtida através do algoritmo de simulação dado no Quadro 1 (seção 2.3), sendo a matriz de correlação teórica $P_{p \times p}$ substituída pela matriz de correlação amostral, ou seja pela matriz $R$ cujos elementos da diagonal principal são iguais a 1, e os elementos que estão fora da diagonal principal são genericamente dados pela Equação 27:

$$
\hat{\rho}_{l j}=\frac{\hat{\sigma}_{l j}}{\hat{\sigma}_{l} \hat{\sigma}_{j}}, l \neq j
$$

sendo $\hat{\rho}_{l j}$ e $\hat{\sigma}_{l j}$ as estimativas da correlação e da covariância entre as variáveis $X_{j}$ e $X_{j}$, e $\hat{\sigma}_{j}$ e $\hat{\sigma}_{j}$ os desvios padrão das variáveis $X_{j}$ e $X_{j}$, respectivamente. Essas estimativas são provenientes da matriz de covariâncias amostral $\bar{S}$ definida pela Equação 23 no caso de subgrupos ou das matrizes dadas pela Equação 24 ou 25 no caso de observações individuais, dependendo do método de estimação usado para a matriz $\Sigma$.

\section{Simulação via Monte Carlo}

Nesta seção apresentamos os resultados de um estudo feito via simulação Monte Carlo com o intuito de avaliar a qualidade de estimação dos índices de capacidade tratados neste artigo. Um total de $\mathrm{k}=1000$ amostras aleatórias de tamanhos $n=50$ e 100 foram geradas considerando-se a distribuição normal bivariada. Os processos simulados foram os casos 4 e 6 definidos na Tabela 2 (seção 5). Para cada amostra aleatória de tamanho $n$ gerada, o vetor de médias do processo foi estimado pelo 
vetor de médias amostral e a matriz de covariâncias do processo pela matriz de covariâncias amostral (S) (Equação 24). A partir dessas estimativas e dos limites de especificação de cada caso, os índices de capacidade foram estimados a um nível de confiança de 99,73\%. Nas Tabelas 6 e 7 são apresentadas as estimativas da média e do desvio padrão da distribuição do estimador do índice de capacidade correspondente assim como o erro médio $(E M)$ e o erro quadrático médio $(E Q M)$, definidos respectivamente pela Equação 28:
$\operatorname{EM}(\hat{\theta})=\frac{1}{k} \sum_{j=1}^{k}\left(\theta-\hat{\theta}_{j}\right)$ e $\operatorname{EQM}(\hat{\theta})=\frac{1}{k} \sum_{j=1}^{k}\left(\theta-\hat{\theta}_{j}\right)^{2}(28)$

sendo $\theta$ o valor verdadeiro do índice de capacidade do processo e $\hat{\theta}_{j}$ a respectiva estimativa obtida com base na j-ésima amostra aleatória de tamanho $n$ gerada no processo de simulação. Os valores teóricos dos índices de capacidade também são apresentados nas tabelas para melhor visualização dos resultados. Todos os estimadores apresentaram boas estimativas. Os maiores erros médio e quadrático

Tabela 6. Resultados médios das estimativas dos índices de capacidade - $n=50$ e 100. Simulação Monte Carlo: processo bivariado caso 4 .

\begin{tabular}{|c|c|c|c|c|c|c|c|c|c|}
\hline \multirow{2}{*}{$\begin{array}{l}\text { Índices de } \\
\text { capacidade }\end{array}$} & \multicolumn{4}{|c|}{$n=50$} & \multicolumn{4}{|c|}{$n=100$} & \multirow{2}{*}{ Valor teórico } \\
\hline & Média & $\mathrm{Dp}$ & EM & EQM & Média & $\mathrm{Dp}$ & EM & EQM & \\
\hline$C_{p}$ - variável 1 & 3,37 & 0,37 & $-0,0404$ & 0,1390 & 3,35 & 0,19 & $-0,0169$ & 0,0352 & 3,33 \\
\hline$C_{p}$ - variável 2 & 2,85 & 0,31 & $-0,0476$ & 0,0977 & 2,82 & 0,16 & $-0,0178$ & 0,0249 & 2,80 \\
\hline$C_{p k}$ - variável 1 & 0,66 & 0,40 & 0,0079 & 0,1568 & 0,68 & 0,30 & $-0,0052$ & 0,0882 & 0,67 \\
\hline$C_{p k}$ - variável 2 & 2,50 & 0,34 & 0,2953 & 0,2042 & 2,56 & 0,20 & 0,2407 & 0,0992 & 2,80 \\
\hline$C_{p}$ (geométrica) & 3,09 & 0,24 & $-0,0393$ & 0,0611 & 3,07 & 0,12 & $-0,0186$ & 0,0149 & 3,05 \\
\hline$C_{p k}$ (geométrica) & 1,21 & 0,44 & 0,1637 & 0,2230 & 1,28 & 0,31 & 0,0929 & 0,1034 & 1,37 \\
\hline$C_{p m A}$ & 0,42 & 0,36 & $-0,0265$ & 0,1278 & 0,42 & 0,27 & $-0,0266$ & 0,0755 & 0,39 \\
\hline$C_{p m B}$ & 0,42 & 0,06 & 0,0076 & 0,0039 & 0,42 & 0,05 & 0,0089 & 0,0023 & 0,43 \\
\hline$C_{p}$ (Niverthi-Dey) & 2,14 & 0,19 & $-0,0084$ & 0,0366 & 2,13 & 0,10 & 0,0007 & 0,0091 & 2,13 \\
\hline$C_{p k}$ (Niverthi-Dey) & $-0,03$ & 0,48 & $-0,0725$ & 0,2330 & $-0,02$ & 0,34 & $-0,0799$ & 0,1239 & $-0,10$ \\
\hline$C_{p m}$ (Mingoti-Glória) & 2,84 & 0,16 & 0,0549 & 0,0292 & 2,82 & 0,10 & 0,0694 & 0,0139 & 2,89 \\
\hline$C_{p m k}$ (Mingoti-Glória) & 0,66 & 0,39 & 0,0292 & 0,1529 & 0,73 & 0,08 & $-0,0383$ & 0,0078 & 0,69 \\
\hline Média - variável 1 & 48,04 & 1,15 & $-0,0364$ & 1,3311 & 47,98 & 0,88 & 0,0162 & 0,7675 & 48,00 \\
\hline Média - variável 2 & 30,06 & 1,16 & $-0,0601$ & 1,3527 & 30,00 & 0,89 & $-0,0039$ & 0,7871 & 30,00 \\
\hline
\end{tabular}

Limites de especificação: variável 1: [30 40 50]; variável 2: [21,6 30 38,4]; Dp: desvio padrão.

Tabela 7. Resultados médios das estimativas dos índices de capacidade $-n=50$ e 100. Simulação Monte Carlo: processo bivariado caso 6 .

\begin{tabular}{|c|c|c|c|c|c|c|c|c|c|}
\hline \multirow{2}{*}{$\begin{array}{l}\text { Índices de } \\
\text { capacidade }\end{array}$} & \multicolumn{4}{|c|}{$n=50$} & \multicolumn{4}{|c|}{$n=100$} & \multirow{2}{*}{ Valor teórico } \\
\hline & Média & $\mathrm{Dp}$ & EM & EQM & Média & $\mathrm{Dp}$ & EM & EQM & \\
\hline$C_{p}$ - variável 1 & 3,38 & 0,36 & $-0,0528$ & 0,1328 & 3,34 & 0,18 & $-0,0108$ & 0,0338 & 3,33 \\
\hline$C_{p}$ - variável 2 & 2,87 & 0,31 & $-0,0714$ & 0,1011 & 2,82 & 0,15 & $-0,0152$ & 0,0234 & 2,80 \\
\hline$C_{p k}-$ variável 1 & 0,68 & 0,39 & $-0,0087$ & 0,1538 & 0,67 & 0,29 & $-0,0030$ & 0,0849 & 0,67 \\
\hline$C_{p k}$ - variável 2 & $-0,55$ & 0,40 & 0,0229 & 0,1601 & $-0,54$ & 0,29 & 0,0065 & 0,0834 & $-0,53$ \\
\hline$C_{p}$ (geométrica) & 3,11 & 0,24 & $-0,0578$ & 0,0593 & 3,06 & 0,12 & $-0,0145$ & 0,0140 & 3,05 \\
\hline$C_{p k}$ (geométrica) & - & - & - & - & - & - & - & - & - \\
\hline$C_{p m A}$ & $-0,45$ & 0,24 & $-0,0275$ & 0,0603 & $-0,46$ & 0,17 & $-0,0170$ & 0,0298 & $-0,48$ \\
\hline$C_{p m B}$ & 0,28 & 0,03 & $-0,0036$ & 0,0011 & 0,28 & 0,02 & $-0,0023$ & 0,0006 & 0,28 \\
\hline$C_{p}$ (Niverthi-Dey) & 2,14 & 0,19 & $-0,0115$ & 0,0350 & 2,13 & 0,09 & 0,0020 & 0,0088 & 2,13 \\
\hline$C_{p k}$ (Niverthi-Dey) & $-0,83$ & 0,47 & 0,0396 & 0,2226 & $-0,80$ & 0,34 & 0,0142 & 0,1136 & $-0,79$ \\
\hline$C_{p m}$ (Mingoti-Glória) & 2,85 & 0,16 & 0,0410 & 0,0276 & 2,82 & 0,09 & 0,0670 & 0,0131 & 2,89 \\
\hline$C_{p m k}$ (Mingoti-Glória) & $-0,55$ & 0,39 & $-0,0013$ & 0,1535 & $-0,54$ & 0,29 & $-0,0121$ & 0,0830 & $-0,55$ \\
\hline Média - variável 1 & 47,99 & 1,14 & 0,0073 & 1,2934 & 47,99 & 0,86 & 0,0147 & 0,7460 & 48 \\
\hline Média - variável 2 & 40,02 & 1,15 & $-0,0175$ & 1,3224 & 40,00 & 0,85 & 0,0012 & 0,7238 & 40 \\
\hline
\end{tabular}

Limites de especificação: variável 1: [30 40 50]; variável 2: [21,6 30 38,4]; Dp: desvio padrão. 
médio ocorreram para a estimativa correspondente ao $C_{p k}$ da variável 2 do processo caso 4 , mas de um modo geral os estimadores apresentaram valores de erro médio próximos de zero, isto é, um baixo vício, e valores de $E Q M$ próximos de zero, ou seja, distribuição com baixa variabilidade em relação aos correspondentes valores teóricos dos índices de capacidade dos processos simulados. Em relação aos dois novos estimadores que estão sendo propostos neste artigo, $C_{p m A}^{M}$ e $C_{p m B}^{M}$, é interessante notar que os resultados foram melhores para o $C_{p m B}^{M}$, que é a extensão do estimador de capacidade de Mingoti e Glória (2008), conferindo a este certa vantagem em relação ao $C_{p m A}^{M}$, que é a extensão proposta para o índice de Niverthi e Dey (2000). Os resultados obtidos nessa simulação indicam também que, em geral, os estimadores $C_{p}^{m}$ e $C_{p k}^{m}$ de Mingoti e Glória (2008) apresentaram melhor desempenho que os índices de Niverthi e Dey $\left(C_{p N D}, C_{p k N D}\right)$. Como esperado, as estimativas foram melhores para $n=100$ em relação a $n=50$.

\section{Intervalos de confiança para os índices de capacidade multivariados}

Intervalos de confiança para o verdadeiro valor dos índices de capacidade multivariados do processo podem ser gerados através da metodologia Bootstrap (RAMOS; HO, 2003). Como ilustração, vamos utilizar os dados apresentados no artigo de Niverthi e Dey (2000), referentes a 50 observações, em centímetros, de 10 características de um componente de um motor de avião. Os respectivos vetores de especificação (de acordo com os autores) são dados pela Equação 29:

$U S L=(6,397 ; 0,600 ; 8,302 ; 7,896 ; 22,051 ;$

$1,856 ; 7,302 ; 6,396 ; 3,052 ; 23,61)^{\prime}$

$L S L=(6,393 ; 0,594 ; 8,294 ; 7,892 ; 22,047$;

$1,852 ; 7,298 ; 6,390 ; 3,038 ; 23,677)^{\prime}$

$\mu^{S}=(6,395 ; 0,597 ; 8,298 ; 7,894 ; 22,049$;

$1,854 ; 7,300 ; 6,393 ; 3,045 ; 23,679)^{\prime}$

Os intervalos de confiança de 95\% para o índice de capacidade real do processo foram construídos a partir da metodologia Bootstrap, utilizando-se 5.000 reamostragens de tamanhos $n=50$. 0 vetor de médias e a matriz de covariâncias $\Sigma$ do processo foram estimados em cada reamostragem para 0 cálculo dos coeficientes de capacidade de acordo com a média amostral e o estimador dado na Equação 24, que é a matriz de covariâncias amostral. Os resultados são mostrados na Tabela 8 , e pode ser observado que todos os índices, com exceção
Tabela 8. Intervalos de 95\% de confiança para o valor real do índice de capacidade multivariado - método Bootstrap.

\begin{tabular}{ccc}
\hline $\begin{array}{c}\text { Índices de } \\
\text { capacidade }\end{array}$ & $\begin{array}{c}\text { Estimativa } \\
\text { pontual (média) }\end{array}$ & $\begin{array}{c}\text { Intervalo de } \\
\text { confiança }(95 \%)\end{array}$ \\
\hline$C_{p}$ (geométrica) & 1,499 & $(1,387 ; 1,631)$ \\
$C_{p k}$ (geométrica) & 1,336 & $(1,239 ; 1,451)$ \\
$C_{p}$ (Niverthi-Dey) & 0,833 & $(0,383 ; 1,272)$ \\
$C_{p k}$ (Niverthi-Dey) & 0,744 & $(0,284 ; 1,172)$ \\
$C_{p}$ (Mingoti-Glória) & 0,766 & $(0,651 ; 0,898)$ \\
$C_{p k}$ (Mingoti-Glória) & 0,711 & $(0,612 ; 0,808)$ \\
$C_{p m A}$ & 0,039 & $(-0,650 ; 0,547)$ \\
$C_{p m B}$ & 0,728 & $(0,644 ; 0,809)$ \\
\hline
\end{tabular}

da média geométrica, indicam que pontualmente o processo é incapaz. Os intervalos de confiança para o verdadeiro índice de capacidade com base nos coeficientes de Niverthi e Dey apontam que o processo pode ser capaz, mas o mesmo não ocorre para os índices propostos por Mingoti e Glória ou as extensões $C_{p m A}^{M}$ e $C_{p m B}^{M}$ propostas neste artigo.

\section{Considerações finais}

Neste artigo, introduzimos dois novos índices de capacidade multivariados, $C_{p m A}^{M}$ e $C_{p m B}^{M}$, que são extensões dos índices univariados $C_{p m}$ e foram construídos com base nas formulações de Niverthi e Dey (2000) e Mingoti e Glória (2008). Mostramos que eles são mais sensíveis a desvios de valores das médias do processo em relação às médias nominais, tornando-se portanto alternativas para avaliação de capacidade de processos multivariados independentes. Deve ser ressaltado que os índices fundamentados na formulação de Niverthi e Dey tendem a apresentar resultados numéricos inferiores aos fundamentados em Mingoti e Glória, podendo até mesmo indicar que um processo é incapaz quando na realidade ele é capaz. Dentro desse contexto, de acordo com os casos tratados neste artigo, o índice $C_{p m B}^{M}$ teve melhor desempenho que o índice $C_{p m A}^{M}$. Os resultados das simulações de Monte Carlo mostraram boa qualidade nas estimativas dos índices teóricos dos processos apresentados neste artigo e indicaram certa superioridade do índice $C_{p m B}^{M}$ em relação ao $C_{p m A}^{M}$ em termos de precisão.

Assim como no caso univariado, é desejável calcular as probabilidades de que o processo gere itens não conformes de acordo com os limites de especificação. No entanto, esses cálculos são mais complexos para processos multivariados a partir de $p=3$. 


\section{Referências}

BERNARDO, J. M.; IRONY, T. X. A general multivariate bayesian process capability index. Statistician, v. 45, n. 3, p. 487-502, 1996.

CHEN, H. A multivariate process capability index over a rectangular solid tolerance zone. Statistica Sinica, v. 4, n. 2, p. 749-758, 1994.

COSTA, F. B.; EPPRECHT, E. K.; CARPINETTI, L. C. R. Controle estatístico de qualidade. São Paulo: Editora Atlas, 2005.

HAYTER, A. J.; TSUI, K. L. Identification and quantification in multivariate quality control problems. Journal of Quality Technology, v. 26, n. 3, p. 197-208, 1994.

JURAN, J. M.; GRYNA, F. M. Controle da qualidade: handbook. São Paulo: Makron, 1992.

KOTZ, S.; JOHNSON, N. L. Process capability indexes-a review, 1992-2000. Journal of Quality Technology, v. 34, n. 1, p. 2-39, 2002.

MASON, R. L.; YOUNG, J. C. Multivariate statistical process control with industrial applications. Pennsylvania: Siam, 2002.

MINGOTI, S. A.; GLÓRIA, F. A. A. Comparing mingoti and glória's and niverthi and dey's multivariate capability indexes. Produção, v. 18, n. 3, p. 598-608, 2008.
MONTGOMERY, D. C. Introdução ao controle estatístico da qualidade. Rio de Janeiro: LTC, 2004.

NIVERTHI, M.; DEY, D. K. Multivariate process capability: a bayesian perspective. Communications in StatisticsSimulation and Computation, v. 29, n. 2, p. 667-687, 2000.

RAMOS, A. W.; HO, L. L. Procedimentos inferenciais em índices de capacidade para dados autocorrelacionados via bootstrap. Produção, v. 13, n. 3, p. 50-62, 2003.

SOARES, A. A. $O$ índice de capacidade multivariado como um instrumento para avaliação do processo de uma operação de usinagem. Dissertação (Mestrado) - Universidade Federal de Santa Catarina, Florianópolis, 2006.

VEEVERS, A. Viability and capability indexes for multiresponse processes. Journal of Applied Statistics, v. 25, n. 4, p. 545-558, 1998.

\section{Agradecimentos}

Agradecemos ao CNPq e à Capes pelo apoio financeiro que possibilitou a execução desse trabalho. Nossos agradecimentos também aos dois revisores anônimos que nos auxiliaram a melhorar a qualidade de apresentação deste artigo.

\title{
Capability indices for independent multivariate processes: extensions of Niverthi and Dey's and Mingoti and Glória's indices
}

\begin{abstract}
In this paper we present two new capability indexes for multivariate independent processes which are extensions of the univariate capability index. These new indexes are based on approach of Niverthi and Dey (2000) and Mingoti and Gloria (2008) and they are sensitive to differences between the specification and the process means. A comparison was performed taking into account several different scenarios which showed that the index based on the Mingoti and Glória approach performed better than the index based on the Niverthi and Dey approach, although both were adequate to evaluate the capability of multivariate non-centered processes. Confidence intervals for the true capability index was built using Bootstrap methodology. The performance of the capability indexes estimators was evaluated by Monte Carlo simulation.
\end{abstract}

\section{Keywords}

Independent multivariate processes. Capability indexes. Multivariate normal. Confidence intervals. Bootstrap. 